\title{
Gravitational constrained instantons
}

\author{
Jordan Cotler $\oplus^{1, *}$ and Kristan Jensen $\oplus^{2,3, \dagger}$ \\ ${ }^{1}$ Society of Fellows, Harvard University, Cambridge, Massachusetts 02138, USA \\ ${ }^{2}$ Department of Physics \& Astronomy, San Francisco State University, \\ San Francisco, California 94132, USA \\ ${ }^{3}$ Department of Physics and Astronomy, University of Victoria, \\ Victoria, British Columbia V8W 3P6, Canada
}

(Received 28 October 2020; accepted 18 August 2021; published 5 October 2021)

\begin{abstract}
We find constrained instantons in Einstein gravity with and without a cosmological constant. These configurations are not saddle points of the Einstein-Hilbert action, yet they contribute to nonperturbative processes in quantum gravity. In some cases we expect that they give the dominant contribution from spacetimes with certain fixed topologies. With negative cosmological constant, these metrics describe wormholes connecting two asymptotic regions. We find many examples of such wormhole metrics and for certain symmetric configurations establish their perturbative stability. We expect that the Euclidean versions of these wormholes encode the energy level statistics of anti-de Sitter black hole microstates. In the de Sitter and flat space settings we find new homogeneous and isotropic bounce and big bang/crunch cosmologies.
\end{abstract}

DOI: 10.1103/PhysRevD.104.L081501

\section{INTRODUCTION}

There has been renewed interest in wormholes stemming from recent progress in low-dimensional quantum gravity. In particular, Euclidean wormholes in Jackiw-Teitelboim (JT) and pure 3D anti-de Sitter (AdS) quantum gravity encode the energy level statistics of black hole microstates in those simple models [1-4]. However, it has been unclear if similar analyses exist in $3+1$ and higher dimensions, where pure quantum gravity requires an ultraviolet completion. At a technical level, it is difficult to find Euclidean wormholes in $d+1 \geq 3$ dimensions which are both perturbatively and nonperturbatively stable. There is a long history of wormhole solutions, nearly all of which are now known to be unstable [5-8]. Furthermore the role of Euclidean wormholes in AdS/CFT is puzzling. If they contribute to the gravity path integral, then there is some tension with the standard holographic dictionary $[6,9]$.

Inspired by recent progress in low-dimensional gravity $[1-4,10-12]$ as well as the resolution of certain information paradoxes via replica wormholes $[13,14]$, we are emboldened to take Einstein gravity seriously as an effective field theory in which one not only considers solutions to the field

\footnotetext{
jcotler@fas.harvard.edu

kristanj@uvic.ca
}

Published by the American Physical Society under the terms of the Creative Commons Attribution 4.0 International license. Further distribution of this work must maintain attribution to the author(s) and the published article's title, journal citation, and DOI. Funded by SCOAP. equations, but a sum over metrics, which we attempt to make sense of as best we can. It is not our purpose nor within our ability to address whether consistent theories of quantum gravity indeed include such a sum; rather, our goal is to mine new physics from the weakly coupled, weakly curved regimes where Einstein gravity ought to make sense as an effective field theory.

In this Letter we find new wormhole configurations in pure Einstein gravity with negative cosmological constant. These wormholes smoothly connect two asymptotic regions, and they are not solutions to Einstein's equations. Indeed, for many cases we study, there are no classical solutions of this sort in pure gravity. Rather, in the language of [15] they are "constrained instantons," meaning that they extremize the Einstein-Hilbert action subject to a constraint. This constraint may be understood to be the length of the wormhole, or the energy perceived by an observer on the boundary. For fixed boundary data there is a $(d+1)$-dimensional family of wormholes, labeled by $d$ "twist" moduli and a parameter that controls the size of the bottleneck of the wormhole. The Einstein-Hilbert action of the wormhole depends on the boundary data and this size parameter.

There is good reason to expect that wormholes in Einstein gravity are constrained instantons. Indeed, the previously studied wormholes in JT gravity and more general 2D dilaton theories [1,2,10-12], as well as pure $\mathrm{AdS}_{3}$ gravity $[3,4]$, are all examples of constrained instantons.

We find a zoo of higher-dimensional Euclidean wormholes, some of which admit a continuation to Lorentzian signature where they become traversable wormholes. These Euclidean metrics admit another continuation to 
new asymptotically de Sitter cosmologies. Taking flat space limits, we find new flat space cosmologies. We discuss these in detail in the Supplemental Material [16]. Further, we initiate a stability analysis of some of the examples. We prove that certain symmetric wormholes are perturbatively stable. Assuming perturbative stability in general, we suggest that the one-loop approximation to the path integral over these instantons (including an integral over the instanton parameters) describes a coarse-grained approximation to the energy level statistics of AdS black hole microstates. In this sense, Euclidean quantum gravity would provide a statistically averaged, "mesoscopic" description of microstates.

\section{CONSTRAINED INSTANTONS}

An essential ingredient in our analysis is the method of constrained instantons [15,17], which we briefly review. We begin with ordinary instantons, to contrast with the constrained case. Given a path integral description of a quantum system, a saddle is a stationary point of the classical action which can be leveraged to perform a saddlepoint approximation of the path integral. An "instanton" usually refers to a localized solution, like the BPST solution of four-dimensional Yang-Mills theory, but we use it to refer to any nontrivial solution. In the $\hbar \rightarrow 0$ limit its contribution to the path integral is weighted by $e^{-S / \hbar}$ with $S$ being the instanton action.

The method of constrained instantons is especially useful in situations where there are no instantons, like a Higgs phase of four-dimensional Yang-Mills theory coupled to matter. To illustrate the idea, consider a Euclidean path integral over a field $\phi$, schematically $\int[d \phi] \exp (-S[\phi] / \hbar)$. For instance, if the theory is a gauge theory, then $S$ includes the classical action, boundary terms, gauge fixing terms, and ghost terms. Let $\mathcal{C}[\phi]$ be some functional of $\phi$. Then

$$
\int[d \phi] e^{-\frac{1}{\hbar} S[\phi]}=\int d \zeta \int[d \phi] \delta(\mathcal{C}[\phi]-\zeta) e^{-\frac{1}{\hbar} S[\phi]},
$$

where we have introduced a constraint $\mathcal{C}[\phi]=\zeta$ but rendered it innocuous by integrating over $\zeta$. As such, the constraint need not be gauge invariant. We can rewrite the above integral as

$$
\frac{1}{\hbar} \int d \lambda \int d \zeta \int[d \phi] e^{-\frac{1}{\hbar}(S[\phi]+\lambda(\mathcal{C}[\phi]-\zeta))}
$$

where $\lambda$ is integrated parallel to the imaginary axis. In the full variational problem one varies with respect to $(\phi, \lambda, \zeta)$ so that the equations of motion are

$$
\delta S[\phi]+\lambda \delta \mathcal{C}[\phi]=0, \quad \mathcal{C}[\phi]=\zeta, \quad \lambda=0,
$$

i.e., $\delta S[\phi]=0$. But suppose we define a new variational problem where one varies $\phi$ and $\lambda$ but keeps $\zeta$ fixed. The corresponding equations of motion are

$$
\delta S[\phi]+\lambda \delta \mathcal{C}[\phi]=0, \quad \mathcal{C}[\phi]=\zeta .
$$

Any solution to the original field equation $\delta S[\phi]=0$ that satisfies the constraint solves these equations. Crucially, this variational problem may admit more solutions. We refer to solutions of (4) with $\lambda \neq 0$ as "constrained instantons." If we have such a constrained instanton then locally in field space it is connected to a one-parameter family of solutions $\left(\phi_{\zeta}, \lambda_{\zeta}\right)$ labeled by $\zeta$. The reason is that perturbing $\lambda$ by a small amount acts as a source in the constrained instanton equations of motion, and these modified equations can then be solved.

Notice that there is a saddle point approximation at fixed $\zeta$; moreover, this is true even when the unconstrained path integral does not admit saddles (e.g., [15]). This leads to a new, candidate semiclassical approximation to the full path integral: we can perform a saddle point approximation at fixed $\zeta$ to some chosen order in perturbation theory, and then integrate over $\zeta$ last. In the semiclassical limit, these saddle point contributions will clearly be important for the path integral evaluation. Let us write a formal expression for the saddle point approximation of the $\left(\phi_{\zeta}, \lambda_{\zeta}\right)$ constrained instantons. To second order in fluctuations, the total action is

$$
\begin{aligned}
S\left[\phi_{\zeta}\right] & +\frac{1}{2} \int d^{d+1} x d^{d+1} y \delta \phi(x) \frac{\delta^{2} S}{\delta \phi(x) \delta \phi(y)} \delta \phi(y) \\
& -i \delta \lambda \int d^{d+1} x \frac{\delta \mathcal{C}}{\delta \phi} \delta \phi .
\end{aligned}
$$

Let $v_{i}$ be the bosonic eigenfunctions of $\frac{\delta^{2} S}{\delta \phi^{2}}$ with eigenvalue $\chi_{i}$, and denote $\int d^{d+1} x \frac{\delta \mathcal{C}}{\delta \phi} v_{i}=\kappa_{i}$. Then the one-loop approximation around the $\left(\phi_{\zeta}, \lambda_{\zeta}\right)$ constrained saddles is

$$
\int d \zeta e^{-\frac{1}{\hbar} S\left[\phi_{\zeta}\right]} V_{\mathrm{zm}}(\zeta) \frac{\mathcal{D}_{F}^{\prime}(\zeta)}{\sqrt{\mathcal{D}_{B}^{\prime}(\zeta)}} \sqrt{\frac{1}{2 \pi} \sum_{i} \frac{\chi_{i}}{\kappa_{i}^{2}}},
$$

where $\mathcal{D}_{B}^{\prime}(\zeta)$ and $\mathcal{D}_{F}^{\prime}(\zeta)$ are the fixed bosonic and fermionic determinants (excepting zero modes) and $V_{\mathrm{zm}}(\zeta)$ is the zero mode volume at fixed $\zeta$.

Below we set $\hbar=1$, but keep factors of Newton's constant $G$ so that $1 / G$ is our large saddle parameter.

\section{CONSTRAINED INSTANTONS FOR EUCLIDEAN AdS}

We begin with Euclidean Einstein gravity in $d+1$ dimensions and negative cosmological constant, with action

$S_{\mathrm{EH}}=-\frac{1}{16 \pi G} \int_{\mathcal{M}} d^{d+1} x \sqrt{g}(R-2 \Lambda), \quad \Lambda=-\frac{d(d-1)}{2 L^{2}}$. 
We impose asymptotically Euclidean AdS boundary conditions and include appropriate boundary terms including those counterterms required by holographic renormalization [18]. Henceforth we use $L=1$ units unless noted otherwise.

The classical solution to Eq. (7) is known: it is just standard Euclidean $\mathrm{AdS}_{d+1}$ with a single boundary. However, our goal is to find a family of constrained instantons which correspond to Euclidean wormholes with two boundaries. We work in a global coordinate system $\left(\rho, x^{i}\right)$ where $i=1,2, \ldots, d$ and $\rho$ is a radial coordinate, and one reaches the two boundaries as $\rho \rightarrow \pm \infty$. We fix $\rho$ so that $g_{\rho i}=0$ and $g_{\rho \rho}=1$. By the logic of Eq. (2), we need to choose an inspired constraint. In this paper we consider two different constraints which lead to the same wormholes. The first constraint is to fix the length of the wormhole connecting the two boundaries as in [12]. This constraint is necessarily noncovariant, and reads

$$
\mathcal{C}\left[g_{\mu \nu}\right]=\frac{1}{8 \pi G} \int d^{d+1} x \Lambda \sqrt{g_{\rho \rho}} F(x),
$$

for a function $F(x)$ to be chosen judiciously later.

The constraint here only depends on $g_{\rho \rho}$, and so only modifies the $\rho \rho$ component of Einstein's equation. In our radial gauge the modified Einstein's equations are

$$
\sqrt{g}\left(R^{\mu \nu}-\frac{R}{2} g^{\mu \nu}+\Lambda g^{\mu \nu}\right)+\lambda F(x) \Lambda \delta_{\rho}^{\mu} \delta_{\rho}^{\nu}=0
$$

Remarkably, we find a wealth of Euclidean wormhole solutions to this constrained problem, many of which we can write down analytically. We focus on a few simple cases, leaving a few more examples to the Supplemental Material [16].

\section{A. Torus boundary}

Consider Euclidean wormholes connecting two regions with torus boundary. These boundaries are specified by independent conformal structures. When these structures are generic and different from each other, we expect to find a wormhole described by a line element of the form

$$
d s^{2}=d \rho^{2}+h_{i j}(\rho) d x^{i} d x^{j},
$$

where the $x^{i}$ parametrize a $d$-dimensional torus. Although we have yet to find a wormhole connecting boundary tori with completely generic conformal structures in all dimensions (we have succeeded in three spacetime dimensions), we have found several special subclasses. The simplest is a highly symmetric configuration which solves the modified equations of motion ${ }^{1}$ :

\footnotetext{
${ }^{1}$ With nonzero $f^{i}(\rho)$ one must modify the constraint slightly so that this metric is a solution of the modified problem.
}

$$
d s^{2}=d \rho^{2}+b^{2}\left(2 \cosh \left(\frac{d \rho}{2}\right)\right)^{\frac{4}{d}} \delta_{i j} d y^{i} d y^{j}
$$

Here $b>0$ and we have relaxed our radial gauge choice slightly by allowing $y^{i}=x^{i}+f^{i}(\rho)$, where $f^{i}(\rho)$ is pure gauge unless it has support out to the boundaries. In that case it contains physical data, a relative twist $\tau^{i}$ between the two boundaries given by $\tau^{i}=\lim _{\rho \rightarrow \infty} f^{i}(\rho)-$ $\lim _{\rho \rightarrow-\infty} f^{i}(\rho)$. This is a bottleneck geometry, with a minimal torus of volume $\sim b^{d}$ at $\rho=0$, so nonsingularity implies $b>0$.

This geometry has two boundaries as $\rho \rightarrow \pm \infty$ with aligned conformal structures specified by the same boundary metric $\delta_{i j}$. By modifying the identifications of the $x^{i}$, we can equip one of the boundary tori with any conformal structure we wish, but then the conformal structure of the other will be aligned with that of the first. Together, the $\left(b, \tau^{i}\right)$ form the instanton parameters at fixed values of the boundary conformal structures. The $\tau^{i}$ are large diffeomorphisms and so exact zero modes, while the renormalized action of this configuration $S_{\text {ren }}=\frac{(d-1) \mathrm{vol}\left(\mathbb{T}^{d}\right)}{2 \pi G} b^{d}$ depends on the size $b$. Figure 1 depicts the wormhole.

The metric (11) satisfies the modified equations (9). It is easy to show that the metric in (11) satisfies the $i j$ and $\rho i$ components of the ordinary Einstein's equations. However, on this metric we find

$$
\sqrt{g}\left(R^{\rho \rho}-\frac{R}{2} g^{\rho \rho}+\Lambda g^{\rho \rho}\right)=\Lambda b^{d}
$$

so that we can solve the $\rho \rho$ component with $\lambda=-b^{d}$ and $F(x)=1$.

In the Supplemental Material [16] we demonstrate two important properties of these wormholes. The first is that they are stable against quadratic fluctuations within Einstein gravity (as well as for more general low-energy effective theories of Einstein gravity coupled to matter). The second is that this is the most general wormhole where the boundary conformal structures are aligned. We show the latter by studying linearized fluctuations of (11) so that the modified line element is of the form (10).

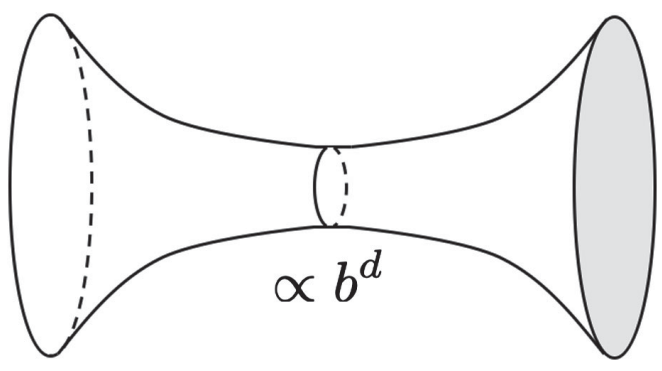

FIG. 1. A schematic of a wormhole with torus boundaries and a bottleneck with volume proportional to $b^{d}$. 
Demanding that the new metric solves the modified Einstein's equations, one finds a $d(d+2)$-dimensional space of perturbations. These perturbations can be understood as changes of the $d+1$ instanton parameters $\left(b, \tau^{i}\right)$, a redefinition $\rho \rightarrow \rho+\varepsilon$, and perturbations in the conformal structures on the two boundaries. There are no other parameters left, which if they existed would parametrize other deformations of the wormhole consistent with the boundary conditions.

We can slightly relax the constraint that the two conformal structures are exactly aligned. A $d$-dimensional torus may be written as $\mathbb{S}_{\beta}^{1} \times_{f} \mathbb{T}^{d-1}$, where the $\mathbb{S}_{\beta}^{1}$ has the interpretation of Euclidean time and the $\mathbb{T}^{d-1}$ as space. Then we may find solutions where the thermal circles on each boundary have different lengths,

$$
\begin{aligned}
d s^{2}= & d \rho^{2}+b^{2}\left(2 \cosh \left(\frac{d \rho}{2}\right)\right)^{\frac{4}{d}}\left(\left(\frac{\beta_{1} e^{\frac{d \rho}{2}}+\beta_{2} e^{-\frac{d \rho}{2}}}{2 \cosh \left(\frac{d \rho}{2}\right)}\right)^{2}\right. \\
& \left.\times\left(d y^{1}\right)^{2}+d s_{\mathbb{T}^{d-1}}^{2}\right),
\end{aligned}
$$

where $d s_{\mathbb{T}^{d-1}}^{2}=\sum_{i=2}^{d}\left(d y^{i}\right)^{2}$ and we let $x^{1} \sim x^{1}+1$. These solutions have a renormalized action

$S_{\text {ren }}=\left(\beta_{1}+\beta_{2}\right) E, \quad E=\operatorname{vol}\left(\mathbb{T}^{d-1}\right) \varepsilon, \quad \varepsilon=\frac{(d-1) b^{d}}{4 \pi G}$,

with $\varepsilon$ the energy density. We will return to this observation shortly.

For the analytic continuation $\beta_{1}=i T$ and $\beta_{2}=-i T$, where the action vanishes, this geometry becomes a genuine saddle for any $b$. This particular configuration is a "double cone" geometry of [1]. Accordingly, for general $\beta_{1}, \beta_{2}$, our configurations generalize the double cone.

Considering (13) for $\beta_{1}, \beta_{2}$ real and positive, analytically continuing $x^{1}$ to real time yields a traversable wormhole connecting the two boundaries at $\rho \rightarrow \pm \infty$ : in this geometry, null geodesics at fixed location on the spatial torus take a finite time to travel from one boundary to the other.

Finally, in addition to the wormhole there is a related singular geometry, with line element

$$
d s^{2}=d \rho^{2}+b^{2}\left(2 \sinh \left(\frac{d \rho}{2}\right)\right)^{\frac{4}{d}} \delta_{i j} d x^{i} d x^{j},
$$

where the whole torus collapses at $\rho=0$ with an "opening angle" $\sim b$. This example will show up later as it admits an interesting continuation to positive cosmological constant.

\section{B. $\mathbb{S}^{1} \times \mathbb{S}^{3}$ boundary}

Let us briefly present another example where the boundaries are $\mathbb{S}^{1} \times \mathbb{S}^{d-1}$, which we expect to be related to black hole physics in AdS. For $d=2$ these boundaries are tori, which we covered above and is given in some more detail in the Supplemental Material [16]. For $d=4$ we find a simple solution with boundaries $\mathbb{S}_{\beta_{1}}^{1} \times \mathbb{S}^{3}$ and $\mathbb{S}_{\beta_{2}}^{1} \times \mathbb{S}^{3}$,

$$
\begin{aligned}
d s^{2}= & d \rho^{2}+\frac{b^{4}}{8} \frac{\left(\beta_{1} e^{2 \rho}+\beta_{2} e^{-2 \rho}\right)^{2}}{b^{2} \cosh (2 \rho)-1}\left(d x^{1}\right)^{2} \\
& +\frac{1}{2}\left(b^{2} \cosh (2 \rho)-1\right) d s_{\mathbb{S}_{3}}^{2},
\end{aligned}
$$

where $x^{1} \sim x^{1}+1$ and nonsingularity implies $b>1$. There are also twist zero modes which we are neglecting to write. In a holographic renormalization scheme where one does not add a finite counterterm $\propto \int d^{4} x \sqrt{\gamma} R_{\gamma}^{2}$, the renormalized action is

$$
S_{\text {ren }}=\left(\beta_{1}+\beta_{2}\right) E, \quad E=b^{4} E_{0}, \quad E_{0}=\frac{3}{8} \frac{\operatorname{vol}\left(\mathbb{S}^{3}\right)}{16 \pi G},
$$

with $E_{0}$ the energy threshold of small black holes in $\mathrm{AdS}_{5}$ in this scheme.

As with the torus wormholes, these metrics describe traversable wormholes upon continuing to real time. Further for $\beta_{1}=-\beta_{2}=i T$ the metric (16) are genuine saddles for all $b$, and reduce to a double cone geometry of [1].

\section{ACTION AND BOUNDARY STRESS TENSOR}

The constrained instantons in the last section have the property that, despite being off shell configurations, their action evaluates to a pure boundary term. In the Supplemental Material [16] we show that this follows from the $i j$ components of the modified Einstein's equations (9), as well as derive a simple expression for the action of those wormholes in terms of the holographic stress tensor $T^{i j}$ [19]. Working in a gauge where our metrics take the form $d s^{2}=d \rho^{2}+h_{i j}(x, \rho) d x^{i} d x^{j}$, the renormalized action for wormholes with torus or $\mathbb{S}^{1} \times \mathbb{S}^{3}$ boundary is

$$
S_{\text {ren }}=\frac{1}{d} \int_{\partial \mathcal{M}} d^{d} x \sqrt{\gamma} \gamma_{i j} T^{i j},
$$

where $\gamma$ is the boundary metric and the action is the sum of two terms, one for each component of the boundary.

We infer that the boundary stress tensor has a nonzero trace for these wormholes. This is in contrast with on shell configurations of Einstein gravity, where the trace of the boundary stress tensor is fixed in terms of the boundary metric [20]. For the torus wormhole in (13) the energy density perceived on the two boundaries is identical and is given by

$$
\varepsilon_{1}=\varepsilon_{2}=\frac{(d-1) b^{d}}{4 \pi G},
$$


while the stress tensor trace on boundary 1 is

$$
\frac{1}{d}\left(\gamma_{i j} T^{i j}\right)_{1}=\frac{(d-1) b^{d}}{8 \pi G}\left(1+\frac{\beta_{2}}{\beta_{1}}\right)
$$

with a similar expression on boundary 2 .

So far we have found constrained instantons by fixing the length between the two boundaries. We would like another, covariant constraint that leads to the same wormholes. Given the boundary stress tensors here, instead we can fix the energies $E_{1}, E_{2}$ perceived on the two boundaries as in $[1,12]$. Indeed our wormholes extremize the EinsteinHilbert action subject to the constraint that $E_{1}=E_{2}=E$, as long as $E$ is above a critical threshold (e.g., the small black hole threshold for the $\mathbb{S}^{1} \times \mathbb{S}^{3}$ case). This likely implies that the wormholes studied in this paper give the dominant contribution to the gravity path integral from spacetimes with this simple topology since we expect that more general wormholes are labeled by fixed $E$, and at least within the subset of metrics of a fixed $E$, our wormholes extremize the action.

\section{STATISTICS OF BLACK HOLE MICROSTATES}

So far we have found families of constrained instantons labeled by the instanton parameters $\left(b, \tau^{i}\right)$. To compute the semiclassical approximation to the path integral as in (6) we require constrained saddles as well as the appropriate oneloop determinants around them. Those determinants may not be tractable for Einstein gravity in more than three spacetime dimensions.

What does the wormhole amplitude encode? In JT gravity and pure $\mathrm{AdS}_{3}$ gravity the full wormhole amplitude has been computed in [2-4]. (In three dimensions the amplitude was computed using a constrain first approach in the first-order formalism [3], rather than by performing a sum over metrics; the amplitude was also computed via a bootstrap method in [4].) In those examples this basic two-boundary wormhole encodes the leading two-point energy fluctuation statistics of black hole microstates, and in particular those statistics match a random matrix theory prediction.

In more than three spacetime dimensions there is much less that we can say without knowing the integration measure over $b$. However, we would like to point out a route by which the one-loop approximation to the wormhole amplitude may yield the same random matrix theory answer as in the lower dimensions, at least in the lowtemperature limit. The torus and $\mathbb{S}^{1} \times \mathbb{S}^{3}$ wormhole actions take the simple form $\left(\beta_{1}+\beta_{2}\right) E$ where $E=V \varepsilon \propto \frac{V b^{d}}{G}$ with $V$ being the spatial volume. (These wormholes also carry zero angular momentum, and so they would encode the spectral statistics of spinless primaries.) Suppose the quantum-corrected measure over $b$ (after integrating out all other fluctuations) is just $d\left(\frac{b^{d}}{G}\right) \sim d \varepsilon$. The integration over the twist zero modes will produce a factor $\mathcal{V}_{0}=\sqrt{\beta_{1} \beta_{2}} V$ (see [3] for a discussion in the context of 3D gravity). We would then have a one-loop amplitude

$$
\sim \mathcal{V}_{0} \int d \varepsilon e^{-\left(\beta_{1}+\beta_{2}\right) V \varepsilon}=\frac{\sqrt{\beta_{1} \beta_{2}}}{\beta_{1}+\beta_{2}} e^{-\left(\beta_{1}+\beta_{2}\right) E_{0}},
$$

where $E_{0}$ is the energy at the lower bound of integration. For a normalization constant $\frac{1}{2 \pi}$ this expression would match a random matrix theory prediction, where $E_{0}$ corresponds to the spinless black hole threshold. This random matrix theory prediction is essentially the physics of level repulsion, which is a generic feature of many-body chaotic quantum systems upon coarse-graining. We cannot help but note that the torus and $\mathbb{S}^{1} \times \mathbb{S}^{3}$ wormholes carry energies $E \geq E_{0}$. Clearly much more work remains to be done.

\section{DISCUSSION}

We have established the existence of constrained instantons in Einstein gravity with and without cosmological constant. With negative cosmological constant, relevant for the AdS/CFT correspondence, we found explicit solutions corresponding to Euclidean wormholes with a variety of boundary topologies. Further, they may give the dominant contribution from two-sided wormholes with trivial bulk topology in pure quantum gravity.

In two and three spacetime dimensions [1-4,21] the statistical properties of AdS black hole microstates are encoded in smooth geometries, albeit constrained instantons (Euclidean wormholes) rather than solutions to the field equations. In JT gravity and pure $\mathrm{AdS}_{3}$ gravity, the two-point fluctuation statistics computed from the simplest wormholes are exactly what one anticipates from a random matrix theory description. It is then striking that there are similar Euclidean wormholes in pure Einstein gravity in $3+1$ and higher spacetime dimensions. With the lower-dimensional results and the universality of random matrix theory in mind, it is tempting to speculate that the path integral over these constrained instantons gives a coarse-grained approximation to the level statistics of AdS black hole microstates. As such, the gravitational path integral would be a mesoscopic description of quantum gravity, which does not know about the precise spectrum of black hole microstates (which in tractable examples depends on the UV completion), but instead provides statistical information.

Recent works have advocated for an ensemble-averaged description of JT gravity and pure gravity in three spacetime dimensions [1-4,21-25], i.e., dualities between pure quantum gravity and a disordered system. JT gravity is renormalizable, and pure $\mathrm{AdS}_{3}$ gravity is power-counting renormalizable, and so modulo the convergence of the sum over topologies, these models do not require a UV completion. In higher spacetime dimension, pure gravity is emphatically nonrenormalizable, but amazingly our low-energy analysis may yield sensible answers for certain statistical quantities probing gravitational microstates. 
We expect that these wormhole amplitudes are akin to the Bekenstein-Hawking entropy, in that they use the effective IR description of quantum gravity to approximate very particular properties of black holes (like the density of states or fluctuation statistics), which naïvely require UV data to reliably compute.

The wormhole metrics that we found are in general macroscopic and smooth, and are within the purview of effective field theory. We caution, however, that curvatures blow up when the bottleneck is small $(b \rightarrow 0$ for the torus wormholes and $b \rightarrow 1$ for the $\mathbb{S}^{1} \times \mathbb{S}^{3}$ wormholes). Nonetheless, outside of this regime, the sum over the wormhole metrics gives a controlled contribution to the gravitational path integral.

We have found that the symmetric $\mathrm{AdS}_{d+1}$ wormholes are perturbatively stable within Einstein gravity, which poses a potential conflict with paradigmic examples of the AdS/CFT correspondence, where certain string theories are equated with single instances of a conformal field theory, rather than an ensemble. With this tension in mind, in [26] we embed the wormholes studied here into string theory in various settings, and perform a stability analysis within perturbative string theory.
There are many future directions suggested by our findings. One is the development of numerical methods for finding constrained instantons, which should be able to leverage existing techniques from numerical relativity. Relatedly, it seems plausible that there exist gravitational constrained instantons with more than two asymptotic boundaries, and perhaps numerical methods may aid in finding these solutions. Another direction is to find constrained instantons for gravity plus matter fields or gauge fields, or even to explore constrained instantons in pure supergravity. Finally, we raised the prospect that perhaps we live in a constrained instanton cosmology. Is this so? It may be useful to find experimental constraints on the instanton parameters, which support the instanton spacetime in a way that mimics cold dark matter.

\section{ACKNOWLEDGMENTS}

We would like to thank D. Stanford for enlightening discussions. J. C. is supported by a Junior Fellowship from the Harvard Society of Fellows. K. J. is supported in part by the U.S. Department of Energy under Grant No. DE-SC 0013682.
[1] P. Saad, S. H. Shenker, and D. Stanford, arXiv:1806.06840.

[2] P. Saad, S. H. Shenker, and D. Stanford, arXiv:1903.11115.

[3] J. Cotler and K. Jensen, J. High Energy Phys. 04 (2021) 033.

[4] J. Cotler and K. Jensen, J. High Energy Phys. 11 (2020) 058.

[5] S. B. Giddings and A. Strominger, Nucl. Phys. B306, 890 (1988).

[6] J. Maldacena and L. Maoz, J. High Energy Phys. 02 (2004) 053.

[7] N. Arkani-Hamed, J. Orgera, and J. Polchinski, J. High Energy Phys. 12 (2007) 018.

[8] T. Hertog, B. Truijen, and T. Van Riet, Phys. Rev. Lett. 123, 081302 (2019).

[9] E. Witten and S.-T. Yau, Adv. Theor. Math. Phys. 3, 1635 (1999).

[10] J. Cotler, K. Jensen, and A. Maloney, J. High Energy Phys. 06 (2020) 048.

[11] E. Witten, Proc. R. Soc. A 476, 20200582 (2020).

[12] D. Stanford, arXiv:2008.08570.

[13] G. Penington, S. H. Shenker, D. Stanford, and Z. Yang, arXiv:1911.11977.

[14] A. Almheiri, T. Hartman, J. Maldacena, E. Shaghoulian, and A. Tajdini, J. High Energy Phys. 05 (2020) 013.

[15] I. Affleck, Nucl. Phys. B191, 429 (1981).

[16] See the Supplemental Material at http://link.aps.org/ supplemental/10.1103/PhysRevD.104.L081501 for additional gravitational constraints instantons in anti-de Sitter, de Sitter, and flat space; a stability analysis of the symmetric torus wormholes; and details of the instanton action and boundary stress tensor.

[17] Y. Frishman and S. Yankielowicz, Phys. Rev. D 19, 540 (1979).

[18] K. Skenderis, Classical Quant. Grav. 19, 5849 (2002).

[19] V. Balasubramanian and P. Kraus, Commun. Math. Phys. 208, 413 (1999).

[20] M. Henningson and K. Skenderis, J. High Energy Phys. 07 (1998) 023.

[21] J. S. Cotler, G. Gur-Ari, M. Hanada, J. Polchinski, P. Saad, S. H. Shenker, D. Stanford, A. Streicher, and M. Tezuka, J. High Energy Phys. 05 (2017) 118.

[22] A. Belin and J. de Boer, Classical Quant. Grav. 38, 164001 (2021).

[23] N. Afkhami-Jeddi, H. Cohn, T. Hartman, and A. Tajdini, J. High Energy Phys. 01 (2021) 130.

[24] A. Maloney and E. Witten, J. High Energy Phys. 10 (2020) 187.

[25] H. Maxfield and G. J. Turiaci, J. High Energy Phys. 01 (2021) 118.

[26] J. Cotler and K. Jensen, J. High Energy Phys. 09 (2021) 001. 